\title{
Study of the morphology of organic ferroelectric diodes with combined scanning force and scanning transmission X-ray microscopy
}

\author{
L. Bernard ${ }^{1 \S *}$, V. Khikhlovskyi ${ }^{2,3 *}$, A. van Breemen ${ }^{3}$, J. J. Michels ${ }^{4}$, R. \\ Janssen $^{2}$, M. Kemerink ${ }^{2,5}$, G. Gelinck ${ }^{2,3}$ and N. Pilet ${ }^{6}$ \\ ${ }^{1}$ Laboratory for Nanoscale Materials Science, Empa, Überlandstrasse 129, 8600 Dübendorf, \\ Switzerland \\ 2 Department of Applied Physics, Eindhoven University of Technology, P. O. Box 513, 5600 \\ MB Eindhoven, The Netherlands \\ ${ }^{3}$ Holst Centre, TNO-The Dutch Organization for Applied Scientific Research, High Tech \\ Campus 31, 5656 AE Eindhoven, The Netherlands \\ ${ }^{4}$ Max Planck Institute for Polymer Research, Ackermannweg 10, 55128, Mainz, Germany \\ ${ }^{5}$ Department of Physics, Chemistry and Biology (IFM), Linköping University, P. O. Box \\ SE-581 83 Linköping, Sweden \\ ${ }^{6}$ Swiss Light Source, Paul Scherrer Institut, CH-5232 Villigen PSI, Switzerland \\ § Corresponding Author: Laetitia.Bernard@empa.ch \\ *Both authors contributed equally to this work.
}

\begin{abstract}
.
Organic ferroelectric diodes attract increasing interest as they combine non-destructive data read-out and low cost fabrication, two requirements in the development of novel nonvolatile memory elements. The macroscopic electrical characteristics and performances of such devices strongly depend on their structural properties. Various studies of their global microscopic morphology have already been reported. Here, a multi-technique approach including different scanning force and X-ray microscopies permitted to reveal and locally study nanometer-scale unexpected sub-structures within a P(VDF-TrFE):F8BT ferroelectric diode. The strong impact of these structures on the local polarizability of the ferroelectric is shown. Two alternative fabrication methods are proposed that prevent the formation of these structures and demonstrate improved macroscopic device performances such as endurance and ON/OFF ratio.
\end{abstract}

Keywords: ferroelectric, organic switch, morphology, scanning force microscopy, X-ray microscopy

This document is the accepted manuscript version of the following article: Bernard, L., Khikhlovskyi, V., Van Breemen, A., Michels, J. J., Janssen, R., Kemerink, M., ... Pilet, N. (2018). Study of the morphology of organic

ferroelectric diodes with combined scanning force and scanning transmission X-ray

microscopy. Organic Electronics, 53, 242-248. http://doi.org/10.1016/

j.orge1.2017.11.012

This manuscript version is made available under the CC-BY-NC-ND 4.0 1icense

http://creativecommons.org/1icenses/by-nc-nd/4.0/ 


\section{Introduction}

Organic ferroelectric resistive switches can be used as nonvolatile memory elements in low-cost organic electronic circuits [1, 2]. They usually consist of a phase-separated blend of ferroelectric and semiconductor polymers sandwiched between two metallic electrodes, combining key properties of the ferroelectrics (non-volatile data storage) and the semiconductor (conductivity/rectification). The resistivity of such diodes can be switched between two or more distinct levels via the control of the stray field emanating from the ferroelectric [3, 4]. These levels retain their state even when the power is turned off. Hence, two-terminal non-volatile memory with non-destructive data read-out can be achieved and up-scaled by using simple crossbar array geometries [5,6].

Macroscopic electrical characteristics of such diodes have been reported [2, 5, 6]. However, it was also shown that the performance of ferroelectric diodes can significantly vary depending on the morphology of the phase-separated blend at the nanoscale, for example arising from different processing conditions [7,8]. The global morphology of such blend films was investigated [8-13], and was shown to consist of semiconducting circular vertical pillar domains embedded in a homogeneous ferroelectric matrix. Some semiconductor domains were found to percolate through the whole matrix layer from bottom substrate to top of the film, enabling proper subsequent electrical contact on both sides. Others semiconductor domains were found to be buried (not reaching the top film surface) or floating (not reaching the bottom substrate). Very recently, additional undesired nanometer-scale structures (called "wetting layer") between bottom contact and a P(VDF-TrFE):F8BT blend have been revealed by selective chemical dissolution [8, 12]. This shows that the film growth processes are not fully under control at lower scale, which can severely impact on the electrical properties and also on the final diodes performance.

Here, a multi-techniques study of a P(VDF-TrFE):F8BT blend is performed to locally investigate at the nanoscale these structures and their influence on the device properties. Local surface and bulk morphological and electrical properties are investigated by a combination of Scanning Transmission X-ray Microscopy (STXM) and Scanning Force Microscopies (SFM), including Kelvin Probe Force Microscopy (KPFM) and Piezo Force Microscopy (PFM). This permitted to determine the nature and understand the origin of these structures. Further, these structures are demonstrated to have a detrimental effect on the local polarizability of

the $\mathrm{P}(\mathrm{VDF}-\mathrm{TrFE})$. Two alternative sample designs are proposed that suppress their formation. This results in a restored homogeneous polarization of the ferroelectric matrix and improved device performances via the increase of endurance and ON/OFF ratio.

\section{Materials and Methods}

The active film of the ferroelectric diodes studied here consists of a 1:9wt/wt blend of poly(9,9-di-n-octylfluorene-alt-benzothiadiazole) - F8BT, and Poly(vinylidene fluoridetrifluoroethylene) - P(VDF-TrFE), with 77 mol\% VDF. Whereas the ferroelectric material $\mathrm{P}(\mathrm{VDF}-\mathrm{TrFE})$ was supplied by Solvay Specialty Polymers, the semi-conductor F8BT was 
synthesized according to a modified Suzuki-polymerization [14, 15]. The blend was spincoated on metal-coated $\mathrm{Si}_{3} \mathrm{~N}_{4}$ membranes (for the combined X-ray/AFM measurements) and glass substrates previously coated/patterned with a metal electrode (for all other measurements), and subsequently annealed at $50^{\circ} \mathrm{C}$ or $135^{\circ} \mathrm{C}$ for $1 / 2$ to 1 hour. The films obtained by this procedure exhibited a thickness of typically $200 \mathrm{~nm}$. For the (patterned) bottom electrode $\mathrm{Au}, \mathrm{OH}$-passivated $\mathrm{Au}$ and $\mathrm{MoO}_{x} / \mathrm{Mo}$ were used. The $\mathrm{Au}$ and Mo contact were obtained by evaporation, followed by the formation of $\mathrm{a} \approx 1 \mathrm{~nm}$ oxide in the case of Mo. The $\mathrm{OH}-\mathrm{Au}$ passivation was performed by a subsequent overnight immersion into a solution of 11mercapto-1-undecanol. The top contact consisted of the SFM tip for all local measurements. For the electrical measurements, a barium electrode capped with aluminium was evaporated on the top of the film.

A schematics of the working principle of the ferroelectric diode is shown in Figure 1 . Since P(VDF-TrFE) is insulating, the current can only flow through the F8BT semiconducting domains. However, an ohmic contact to the electrodes is needed to inject charges. The bottom electrode material was purposely chosen to form a Schottky barrier at the interface with F8BT, insuring a limited charge injection and a low current flow. When the ferroelectric is polarized along the direction of the probing bias (Figure $1 \mathrm{~A}$ ), the emanating stray field increases the apparent injection barrier and fully suppresses the current in the diode (OFFstate). In contrast, when the ferroelectric is inverse-polarized with respect to the probing bias (Figure 1B), the stray field (curved dashed black arrows) lowers the apparent injection barrier at the semiconductor/metal interface and a higher diode current is observed (ON-state) [2, 3]. The typical hysteretic loop between ON- and OFF-state of the diode is shown in Figure $1 \mathrm{C}$, with $V_{c}$ being the minimum voltage to inverse-polarize the ferroelectric (coercive voltage).
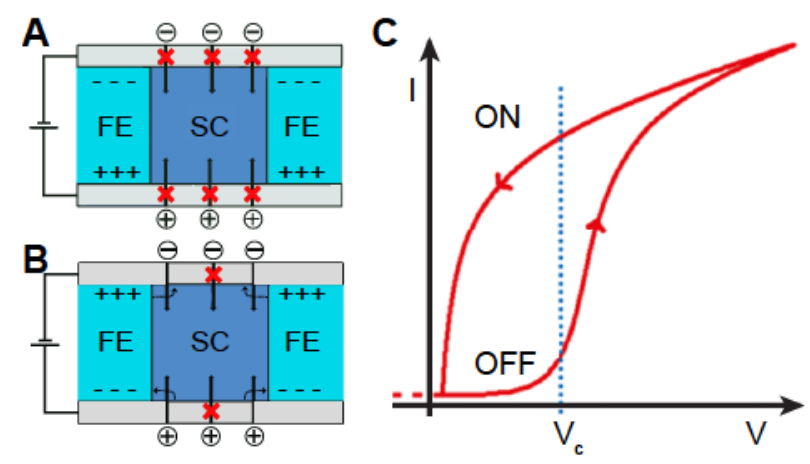

Figure 1. A./B. Cross-sectional schematics of the diode, with the ferroelectric (FE, cyan) and the semiconductor (SC, blue) domains, top and bottom electrodes (grey), in the OFF-state (polarization and bias voltage with same polarity) and $\mathrm{ON}$-state (polarization and bias voltage with inverse polarity), respectively. Polarization charges in the ferroelectric, direction of the stray field (black dashed arrows) and charge carriers transport in the semiconductor (black arrows) are indicated. C. Typical IV characteristics of a Au/P(VDF-TrFE):F8BT/Au diode, being switched in the ON-/OFF-state by applying bias with amplitude exceeding the coercive voltage $V_{c}$ of $\mathrm{P}(\mathrm{VDF}-\mathrm{TrFE})$.

Kelvin force microscopy was carried out on a prototype SFM instrument developed by 
Empa. Measurements were performed in ultra high vacuum at $\approx 10^{-8} \mathrm{mBar}$, with a Pt-coated tip from Nanosensors (PPP-ContPt). A phase-locked loop was used to drive the cantilever on its resonance of $12.9 \mathrm{kHz}$ with an oscillation amplitude kept constant. To measure the Kelvin signal an oscillatory bias of $100 \mathrm{mV}$ with a frequency of $9.7 \mathrm{kHz}$ was applied, and the resulting oscillatory cantilever deflection was detected with a lock-in amplifier and minimised by adding a DC voltage compensating exactly the local surface potential. Note that since the surface potential originates from the electronic properties of material extending over several hundreds of nanometers in the depth, the full polymer film thickness is expected to contribute to the KPFM signal.

The combined X-ray/AFM data was measured at the NanoXAS beam-line of the Swiss synchrotron (SLS), Paul Scherrer Institute [16]. Measurements were performed in ultra high vacuum at $\approx 10^{-9} \mathrm{mBar}$. X-ray data were acquired in transmission mode at energy much higher than the carbon resonance $(1 \mathrm{keV})$. The STXM data presented here show the absorbed $\mathrm{X}$-ray intensity $\left(I_{0}-I_{\text {transmitted }}\right)$, such that dark reflects low absorption and bright strong absorption. Thus, the STXM intensity predominantly reflects the film density and thickness. The topography was measured by AFM operated in contact mode with a Pt-coated cantilever from Nanosensors (PPP-ContSCPt).

The poling of the $\mathrm{P}(\mathrm{VDF}-\mathrm{TrFE})$ matrix and piezo force microscopy study was achieved with an hrMFM instrument (NanoScan) at Empa. Poling and measurements were performed in contact mode operated in air. A Ti/Ir-coated AFM tip from Asylum Research (ASYELEC) with a contact resonance of $\approx 300 \mathrm{kHz}$ was used to map the topography, pole the ferroelectric matrix by scanning it in contact with the blend [23] with a DC bias of $\pm 20 / 25 \mathrm{~V}$ - well above the coercive voltage of the $\approx 200 \mathrm{~nm}$-thick $\mathrm{P}(\mathrm{VDF}-\mathrm{TrFE})$ films $(\approx 10 \mathrm{~V}$ according to measured piezoresponse loops [24]), and to measure the PFM phase and amplitude maps. For the latter a lock-in amplifier from Zürich Instruments was used.

\section{Results and Discussion}

\subsection{Nature of the undesired structures}

A typical P(VDF-TrFE):F8BT blend formed on Au substrate has been investigated by a combination of AFM and KPFM. Figure 2A shows the topography of the blend, acquired by AFM operated in non-contact mode. One can distinguish the F8BT semiconducting circular domains within the $\mathrm{P}(\mathrm{VDF}-\mathrm{TrFE})$ ferroelectric matrix, as already reported in previous work on this blend [8, 12]. However here, all F8BT domains appear to protrude from the matrix surface. Moreover, additional unexpected structures, mostly visible as "cressant-like halos" surrounding the F8BT domains, are visible. Various effects can lead to this observation. Additional material of either types might have accumulated at the F8BT/P(VDF-TrFE) interface due to surface tension or capillary forces. On the other hand, because $\mathrm{P}(\mathrm{VDF}-\mathrm{TrFE})$ is insulating, a charge accumulation might have occurred at the interface, leading to increased electrostatic force around the F8BT domains that in turn appears brighter. To elucidate this, KPFM measurements were performed on the same area with a locally compensated contact 
potential. Note that in contrast to conductive AFM (c-AFM) - where only fully percolating structures/domains can be detected via the measurement of the current flowing through them, KPFM probes the electrostatic interaction between tip and sample, that typically spans over several hundreds of nanometers $\left(\approx 1 / d^{2}\right)$. Hence, electrostatic contribution from the whole film thickness - including local charges, even if very localized, is expected to be detected.

Figure $2 \mathrm{~B}$ and $\mathrm{C}$ present the corresponding topography - this time free of possible charge effects, and contact potential maps, respectively. Comparing the topography images without (A) and with (B) KPFM feedback reveals significant differences. The F8BT domains no longer appear so clearly protruding; two of them (see red arrows) even appear buried. The crescent-like structures surrounding the cylindrical semiconducting domains still appear to protrude in the topography image. This demonstrates that they are due to excess of material, and not to the presence of local charges. From Figure $2 \mathrm{~B}$, one can estimate their lateral spread to $\approx 600 \mathrm{~nm}$-wide, radial to each F8BT domain, and their thickness to range from few tens of nanometers up to more than $100 \mathrm{~nm}$. The contact potential image (C) shows very bright circular areas that correspond to the F8BT domains observed in the topography images. Note that all these domains, including the buried ones, exhibit the same contact potential ( $\approx 160 \mathrm{mV}$ with respect to that of the $\mathrm{P}(\mathrm{VDF}-\mathrm{TrFE})$ matrix $)$, as visible on the crosssection ( $\mathrm{D}$, corresponding to the blue line). The crescent-like structures are also visible, with a much lower intensity compared to that of the F8BT domains but larger than that of the $\mathrm{P}(\mathrm{VDF}-\mathrm{TrFE})$ matrix. From this, it appears clear that the material of the structures can neither be an excess of P(VDF-TrFE) - what would imply no or negligible change in intensity, nor F8BT at the surface - what would appear almost as bright as the F8BT domains themselves. This demonstrates that the structures must be composed of buried F8BT, presumably located at the blend/Au interface.

\subsection{Cause for the undesired structures formation}

In order to understand the origin of formation of the undesired structures and find strategies to suppress them, combined STXM / AFM measurements have been performed on three samples with different interface configurations. AFM topography images were acquired at the same sample locations as the STXM data to insure a correct interpretation of the X-ray images. The first sample consists of the blend directly deposited on Au and annealed at $135^{\circ} \mathrm{C}$ for 1 hour, similarly to the sample of Figure 2. The second sample is of similar composition but annealed at a lower temperature $\left(50^{\circ} \mathrm{C}\right)$ and for $1 / 2$ hour only ("low-T-annealed"). The third sample is similar to the first one, except that the Au surface was passivated with $\mathrm{OH}$-terminated SAM prior to the blend deposition. Figure 3 shows the AFM topography and the corresponding STXM images for the three samples. On the first sample (A), the observed topography is similar to that shown in Figure $2 \mathrm{~B}$, showing protruding and buried F8BT circular domains, and the surrounding structures. Surprisingly, the F8BT domains are not visible in the X-ray absorption image. Instead, structures much larger than the F8BT domains become visible. Sample B (annealed at lower temperature) shows a substantially different morphology: First, the surrounding structures are difficult to distinguish from the matrix background. Moreover, 


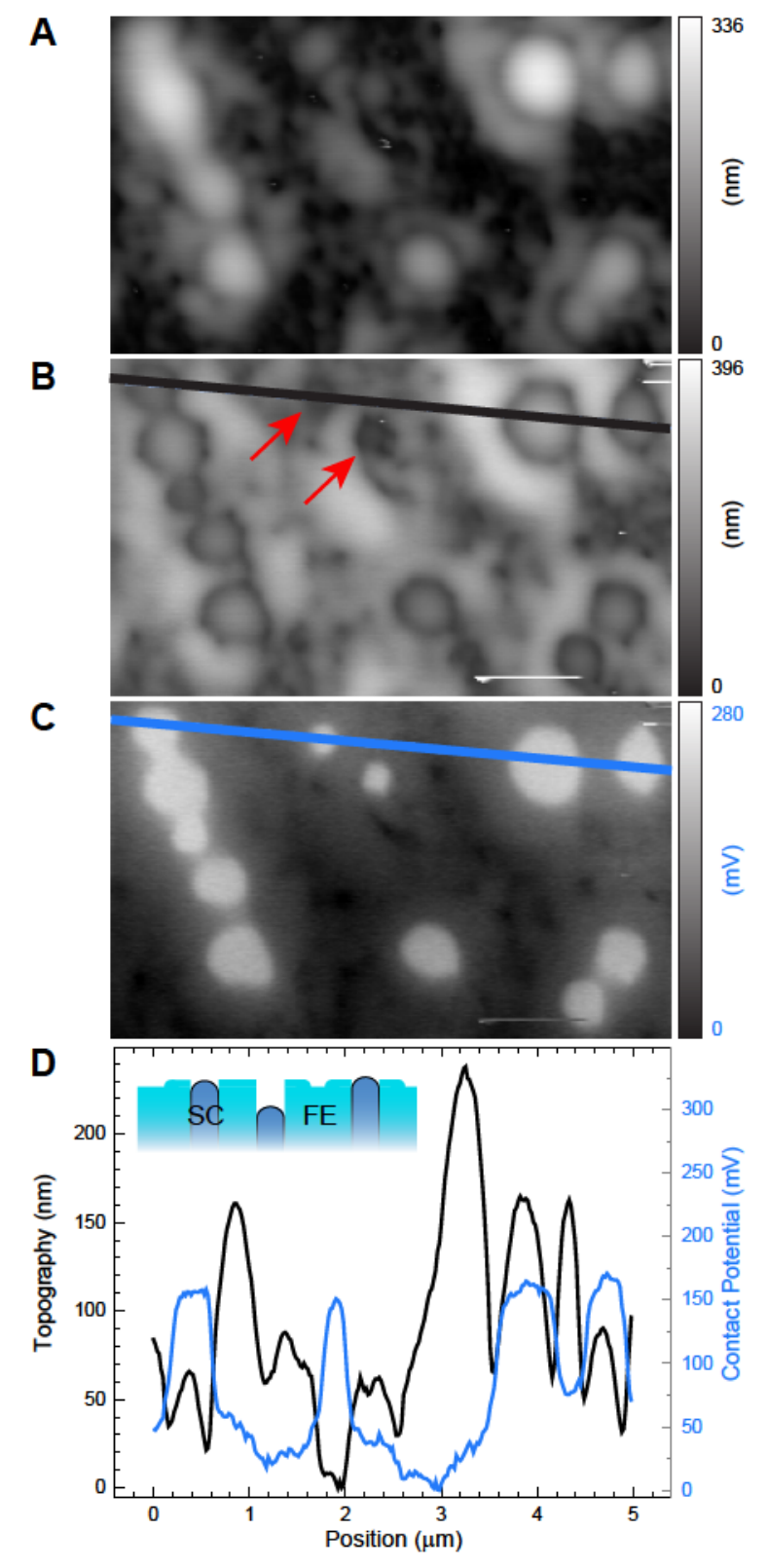

Figure 2. KPFM study of a P(VDF-TrFE):F8BT blend deposited on Au substrate, $5 \times 3 \mu \mathrm{m}^{2}$ : A. Topography image without KPFM feedback; B. Topography image with KPFM feedback; C. Corresponding contact potential image; D. Cross-sections of B. and C. along the indicated black/blue line respectively. The insert scheme depicts the cross-sectional conformation of the film close to the surface.

needle-like structures - a well-known structure of P(VDF-TrFE [18, 19], are visible. In spite of the differences between the topography and x-ray images, F8BT domains are visible in both data sets. In the x-ray image, the F8BT domains appear dark because F8BT is of lower density than $\mathrm{P}(\mathrm{VDF}-\mathrm{TrFE})[20,21]$. The variation of the contrast in the $\mathrm{P}(\mathrm{VDF}-\mathrm{TrFE})$ matrix can be explained by spatial variations of the thickness, or arises from the presence of the crystallites that are surrounded by an lower density amorphous phase. On the $\mathrm{OH}$-passivated 
sample (C), the diameter of the F8BT domains are even smaller than those of samples A and B. Consequently a higher density is observed. This substantial change of the sample morphology can be explained by the preferential adsorption of $\mathrm{P}(\mathrm{VDF}-\mathrm{TrFE})$ on $\mathrm{OH}$-passivated Au due to its much lower surface energy as compared to that of F8BT [8]. The X-ray image corresponds well with the topography, exhibiting the F8BT domains as dark circular areas and a significantly more homogeneous bright $\mathrm{P}(\mathrm{VDF}-\mathrm{TrFE})$ matrix. No larger surrounding structures are observed in the X-ray image as opposed to the non-passivated sample (A). This demonstrates that the formation of the crescent-like F8BT structures surounding the cirular domains is dependent on the blend/substrate interaction, and confirms that the structures form at the interface. Further, their formation can be avoided by the presence of the OH-terminated SAM at the interface. This strongly suggests that the formation of such structures at the blend/Au interface is driven by a strong coordinative interaction of F8BT with Au, as expected to arise from the formation of S-Au bonds [8,22].
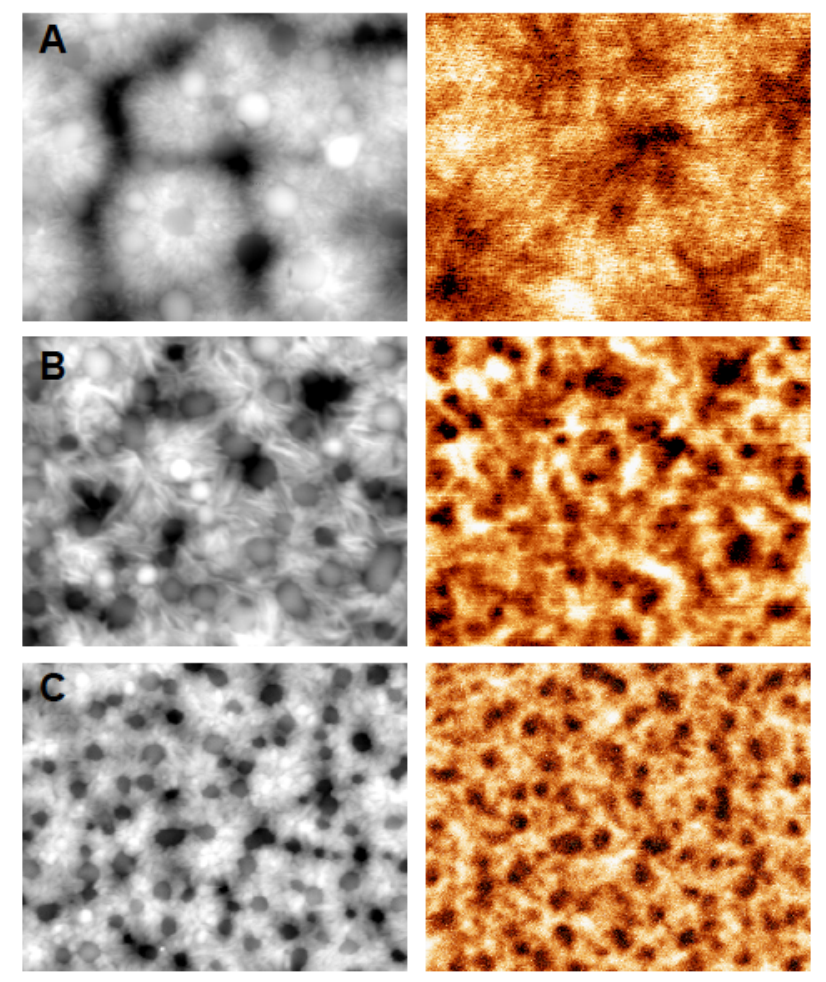

Figure 3. Combined SFM/STXM study, topography (left) and X-ray intensity (right), of a P(VDF-TrFE):F8BT blend deposited on: A. Au substrate (normal annealing), B. Au substrate with low-T-annealing and C. OH-passivated Au substrate (normal annealing). FOV $5 \times 4 \mu \mathrm{m}^{2}$.

\subsection{Effect of the undesired structures on the polarizability and diode performances}

Since a strong modulation of the injection barrier of the diode requires a maximal ferroelectric polarization [3], the effect of the undesired structures on the polarizability of $\mathrm{P}(\mathrm{VDF}-\mathrm{TrFE})$ was studied by PFM. This method locally maps the piezoelectric action of the material that is 
a direct measure for the materials polarization state. Figure 4 shows the topography (left) and piezo-amplitude (center-left) of a blend/Au sample after positive (A) and negative (B) poling. Note that poling areas of $8 \times 8 \mu^{2}$ were chosen concentric but smaller than the imaging areas $\left(10 \times 10 \mu \mathrm{m}^{2}\right)$ to make the change of the piezoelectric motion before and after poling visible. The topography of the sample observed in these conditions is similar to that of the previous SFM measurements: protruding and buried F8BT circular domains within the P(VDF-TrFE) matrix are visible, and also the undesired crescent-like F8BT structures. For positive poling (positive pole at the top surface), the piezo-amplitude image (Figure 4 A, center-left) shows dark areas with a diameter that is much larger than the F8BT domains visible in the topography image (Figure $4 \mathrm{~A}$, left). This demonstrates that in this case, only the P(VDF-TrFE) outside the surrounding crescent-like F8BT structures contribute to the observed piezo-response signal. The piezo-response data obtained after negative poling however appears very different (Figure $4 \mathrm{~B}$, center-left). There, the diameters of the dark areas observed in the piezo-response image match those of the F8BT circular domains in the topography image (Figure $4 \mathrm{~B}$, left). These two observations can be explained by considering the two poling situations at the undesired structures location.

Figure $4 \mathrm{C}$ shows a cross-sectional scheme of a semiconducting domain including the undesired structure at its bottom (center-right) and poling schematics at the location of the undesired structure for both positive (top) and negative (bottom) poling polarities, respectively (right). Upon the application of a positive poling bias (Figure $4 \mathrm{C}$, top), charges accumulate via the contacts at both sides of the blend, creating the electrical field needed to pole the ferroelectric. However, a ferroelectric/semiconductor bilayer that acts as two capacitors in series is present. In addition, a Schottky barrier at the electrode/semiconductor interface is present and is biased in the reverse current state (injection barrier is high). The bias voltage is thus divided, with most of the voltage drop occuring in the semiconductor. The remaining voltage at the ferroelectric boundaries is much lower than initially intended and the resulting electrical field remains below the coercive field [25-27]. As a consequence, no polarization and in turn no piezo-amplitude can be obtained - and thus observed - at these locations. Under negative poling though (Figure $4 \mathrm{C}$, bottom), the structures are not expected to have any influence on the poling process. F8BT being a p-type semiconductor, the system is poled in the forward direction, and the contact can provide holes to the semiconductor. The semiconductor under negative poling thus acts as an extension of the bottom electrode. The bias voltage drop in this case fully occurs over the ferroelectric, leading to an electrical field of about $100 \mathrm{MV} / \mathrm{m}$, i.e. far above the $\mathrm{P}(\mathrm{VDF}-\mathrm{TrFE})$ coercive field of $50 \mathrm{MV} / \mathrm{m}$ [18]. Hence, a proper poling of $\mathrm{P}(\mathrm{VDF}-\mathrm{TrFE})$ is obtained, even at the location of the undesired structures.

Even if occurring for positive poling only, the effect of the F8BT structures on the polarizability of $\mathrm{P}(\mathrm{VDF}-\mathrm{TrFE})$ as revealed in this PFM study can have important consequences since the ON-/OFF-state switching of the diode relies on the charge injection from the polarized ferroelectric into the semiconducting domains. If P(VDF-TrFE) is not polarized at the ferroelectric/semiconductor interface, a strong reduction of the injection effect is to be expected. The STXM / AFM study demonstrated that the formation of these structures could be prevented by using a gold-passivated substrate. 

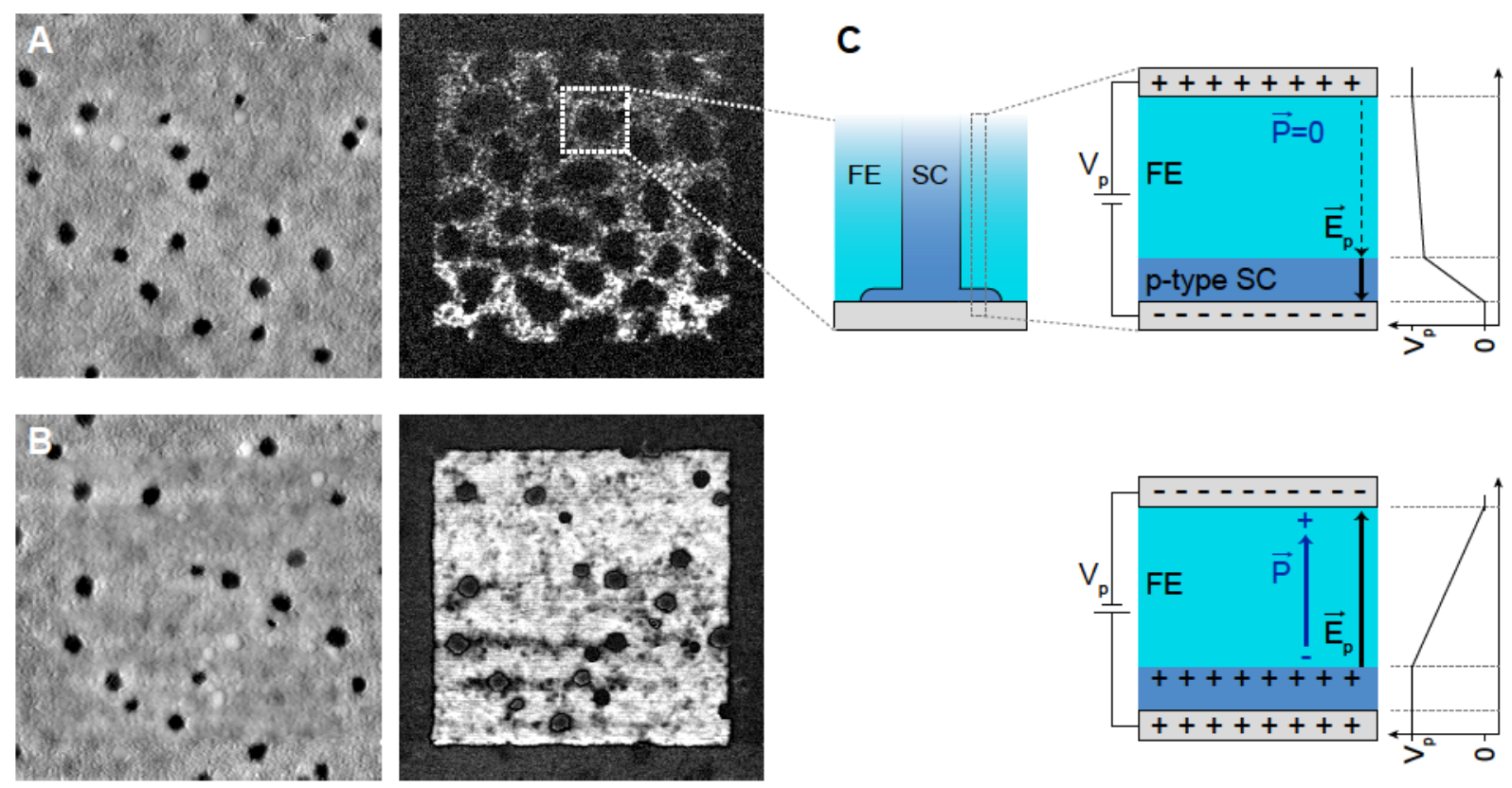

Figure 4. Topography (left) and piezoforce-amplitude (center-left) PFM images of a P(VDFTrFE):F8BT blend deposited on Au electrode, under A. $V_{p}=+20 \mathrm{~V}$ poling (downwards polarization) and B. $V_{p}=-20 \mathrm{~V}$ poling (upwards polarization). Image FOV: $10 \times 10 \mu \mathrm{m}^{2}$; concentric $8 \times 8 \mu \mathrm{m}^{2}$ poling area. C. Cross-sectional scheme of the film at the location of a crescent-like structure, close to the interface with the bottom electrode (left) and poling schematics (right) corresponding to the respective $+20 \mathrm{~V}$ and $-20 \mathrm{~V}$ poling. For positive $V_{p}$, the resulting poling electrical field $\left(\vec{E}_{p}\right)$ is lower than the coercive field due to bias drop at the Schottky barrier; the ferroelectric thus cannot be polarized $(\vec{P}=0)$.

Here, a similar but gold-free sample with molybdenum $\left(\mathrm{MoO}_{x} / \mathrm{Mo}\right)$ as bottom electrode, was investigated. Note that in addition to reducing the coordinative interactions with the substrate, $\mathrm{MoO}_{x}$ is a transition metal oxide that is increasingly used in polymer-based electronics due to its high work function, good ohmic contact and hole injection properties [28, 29]. Figure 5 shows PFM topography (left) and piezo-amplitude (center-left) images of the blend/ $/ \mathrm{MoO}_{x} / \mathrm{Mo}$ sample, under positive (A) and negative (B) poling. Similarly to the $\mathrm{OH}$-passivated Au-based sample, the morphology of the blend is found to present a larger density of F8BT circular domains with significantly smaller diameter. In contrast with the images obtained on the Au-based sample (Figure 4), none of the piezo-amplitude images reveal any sign of crescent-like structures. This demonstrates that with $\mathrm{MoO}_{x} / \mathrm{Mo}$ as substrate, the undesired structures do not form and the semiconductor/ferroelectric blend has the desired conformation (Figure $5 \mathrm{C}$, center-right). This in turn allows an even poling of the $\mathrm{P}(\mathrm{VDF}-\mathrm{TrFE})$ matrix with both polarities, including in the close vicinity of the F8BT domains (Figure 5 C, right).

Besides the qualitative similarity between positively and negatively poled areas, it appears that the piezo-amplitude significantly varies in intensity. The average piezo-amplitude values in the $8 \times 8 \mu \mathrm{m}^{2} \mathrm{P}$ (VDF-TrFE) poled areas were extracted from the piezo-images of Figures $4 \mathrm{~A}$ and $\mathrm{B}$ and $5 \mathrm{~A}$ and B. Note that F8BT domains and undesired crescent-like 
structures in the case of Au-based sample were excluded. Piezo-amplitude of $37 / 174 \mathrm{mV}$ for positive/negative poling of the Au-based sample, and of 143/423 mV of the $\mathrm{MoO}_{x} / \mathrm{Mo}$-based sample, respectively, are observed. Based on those values, two major trends can be identified. First, negative poling appears significantly more efficient than positive poling, with factors of 4.7 and 2.9 difference for $\mathrm{Au}$ and $\mathrm{MoO}_{x} / \mathrm{Mo}$ electrode material, respectively. Similar preferential polarization of $\mathrm{P}(\mathrm{VDF}-\mathrm{TrFE})$ upon negative poling has already been evidenced by pyroelectric investigation [30], where more efficient charge injection at the bottom electrode was hypothesised. Here, the presence of a very thin layer of non-ferroelectric material at the interface is also likely to play a role in this large asymmetry. The interface configuration leads to a facilitated injection from the bottom electrode upon negative poling (Figure $4 \mathrm{C}$, bottom). A second remarkable observation is that using $\mathrm{MoO}_{x}$ /Mo electrode leads to a larger piezoeffect in the P(VDF-TrFE) matrix than with a gold electrode, by factors of 4.0 and 2.4 for positive and negative poling, respectively. Note that a slightly larger poling voltage was used for the $\mathrm{MoO}_{x} / \mathrm{Mo}$-based sample $(25 \mathrm{~V}$ instead of $20 \mathrm{~V})$, but both voltages were significantly larger than the coercive voltage, in principle insuring a maximal polarization in both cases. A tentative explanation for the observed increased polarizability with $\mathrm{MoO}_{x} / \mathrm{Mo}$ electrode could be a morphology change of the ferroelectric $\beta$-phase of P(VDF-TrFE). Whereas P(VDF-TrFE) ferroelectric crystallite growth can be promoted by thermal treatments such as annealing [19] or X-ray irradiation [31], the steric constraints at the substrate interface can act on the mobility and interaction between the different polymer phases and thus on the growth of the crystallites. Also, the substrate surface energy was shown to influence the orientation of the crystallites for thin films [32]. A better alignment of the crystallites parallel to the field (edgeon) would increase the global polarizability perpendicular to the substrate and consequently also the piezo-amplitude as measured here. A difference in the electromechanical coupling between the intermixed crystallites and amorphous phase [33] or a more efficient $\alpha$ - to $\delta$-phase transformation upon poling [34] could also contribute to this increased piezo-effect.

In the light of the polarization results, the impact of the undesired crescent-like structures on the final diode performance was investigated by cyclic electrical measurements. Figure 6 shows the current versus voltage characteristics upon consecutive program-erase cycles in BaAl/P(VDF-TrFE):F8BT/Au (A) and BaAl/P(VDF-TrFE):F8BT/MoO $/$ /Mo (B) diodes, showing typical ferroelectric junction switching behavior [2]. The current at voltages below the coercive voltage is low, because of the high injection barrier at the bottom contact in the un-poled state of the ferroelectric. With bias voltage increasing above $V_{c}$ (see arrows), the $\mathrm{P}(\mathrm{VDF}-\mathrm{TrFE})$ matrix becomes upwards polarized, leading to an effective lowering of the hole injection barrier at the bottom electrode, while the top electrode barrier remains high [3]. Current settles in the F8BT domains and the diode turns in the ON-state. Upon bias voltage decrease, the $\mathrm{P}(\mathrm{VDF}-\mathrm{TrFE})$ polarization remains upwards, and hence the device remains in the ON-state that is characterized by a high forward current, until $-V_{c}$ is reached. At that moment, the polarization switches downwards, lowering this time the injection barrier at the top electrode and restoring the current in the F8BT. However, the current remains much lower than for positive bias, because the top electrode material $(\mathrm{BaAl})$ was purposely chosen to act as blocking electrode (low hole injection). The diode thus remains in the OFF-state upon the 

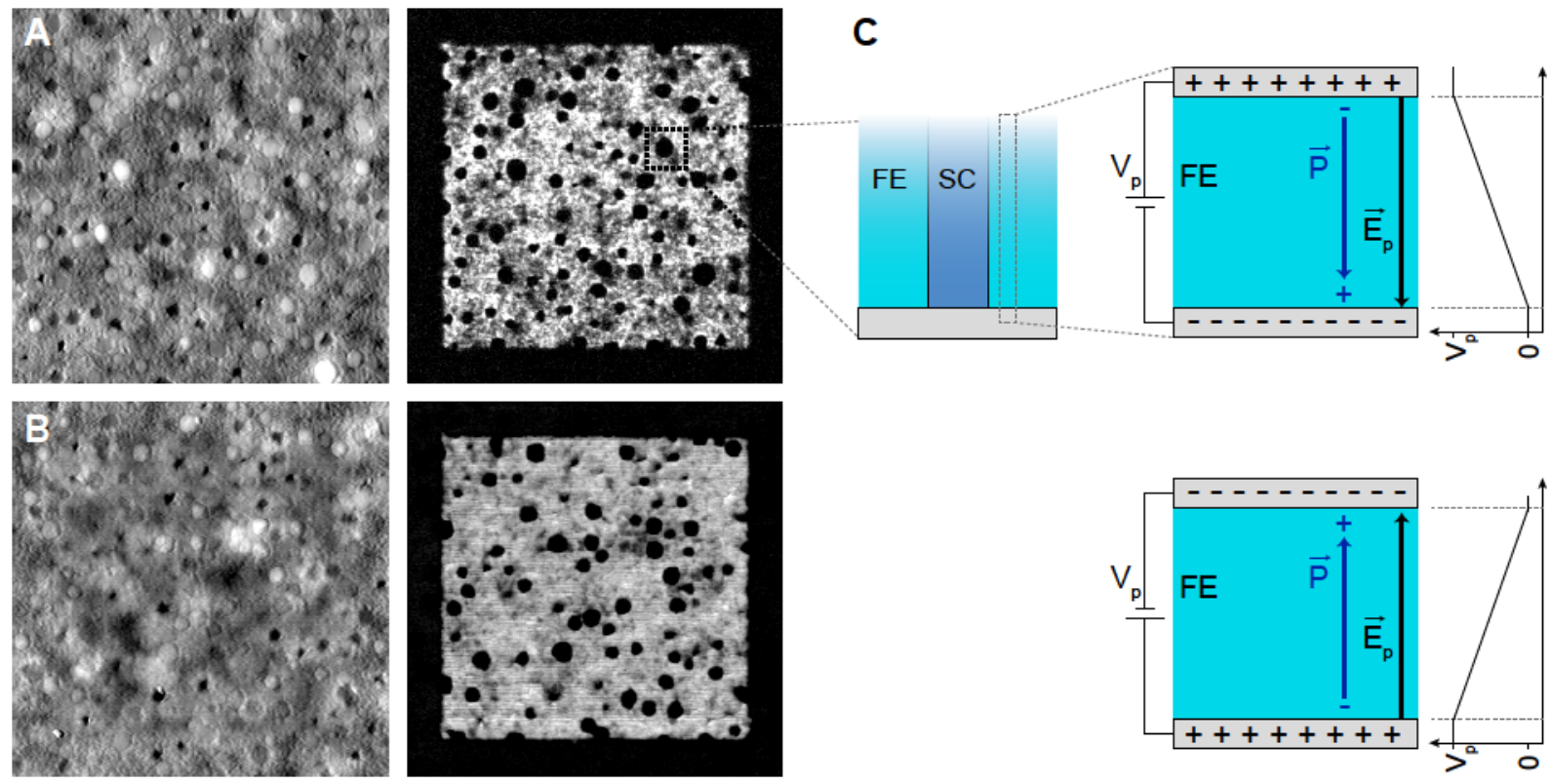

Figure 5. Topography (left) and piezoforce-amplitude (center-left) PFM images of a P(VDFTrFE):F8BT blend deposited on $\mathrm{MoO}_{x}$ electrode, under A. $V_{p}=+25 V$ poling (downwards polarization) and B. $V_{p}=-25 V$ poling (upwards polarization). Image FOV: $10 \times 10 \mu \mathrm{m}^{2}$; concentric $8 \times 8 \mu \mathrm{m}^{2}$ poling area. C. Cross-sectional scheme of the film close to the interface with the bottom electrode (left) and poling schematics (right) corresponding to the respective $+25 \mathrm{~V}$ and $-25 \mathrm{~V}$ poling.

whole loop of negative biasing. The bias is then increased again towards positive values and a new cycle is started. The endurance of the ferroelectric switching junction, i.e. the evolution of the I-V characteristic upon many cycles repetition has been studied. For the gold-based diode (Figure 6A), a continuous reduction of the maximum current (corresponding to the ONstate) is observed between 1 program-erase cycle (black curve), 10 (red curve), 100 (blue curve) and 1000 (purple curve) cycles. The latter shows a decrease of more than one order of magnitude as compared to the initial performances (first cycle).

The effect of the undesired crescent-like structures is investigated by comparing the I$\mathrm{V}$ characteristics of the $\mathrm{MoO}_{x} / \mathrm{Mo}$-based diode (Figure 6B) with that of the gold-based diode. No significant difference can be observed in the current amplitudes of the ON- and OFF-states for positive biases. This was to be expected since the bottom electrode is in this case positively charged, thus injecting holes. This brings the diode in the state of Figure $4 \mathrm{C}$, bottom, where the undesired structures have no detrimental influence on the polarizability. In the reversed bias polarity however, the bottom electrode is negatively charged and the undesired structures are expected to have an influence on the charge injection. In Figure 6, one sees that at negative bias the maximum current obtained with $\mathrm{MoO}_{x}$ /Mo-based diode is one order of magnitude larger than that of the gold-based diode. This confirms that the presence of these structures does have a significant detrimental influence on the charge injection. However, in the present situation, that effect is rather beneficial, since a minimal current is sought for negative biasing.

On the other hand, the cycling test demonstrates very different endurance properties. 
While the gold-based diode shows a decrease of the ON-state current of more than one order of magnitude after 1000 cycles, that of the $\mathrm{MoO}_{x} / \mathrm{Mo}$-based diode is mostly constant upon the different numbers of program-erase cycles. The endurance thus appears to be greatly improved by using $\mathrm{MoO}_{x} / \mathrm{Mo}$ bottom electrode instead of $\mathrm{Au}$. One possible explanation for the degraded endurance of the Au-based device as compared to the Mo-based one is the effect of the extremely high field over this very thin interlayer (see Figure $4 \mathrm{C}$ ). This field could have induced an uncontrolled degradation in the semiconductor, leading to a local loss of its semiconducting properties. The sample could have developed the same problems in the forward direction as in the reverse(see Figure $4 \mathrm{C}$, top), leading to a reduced supply of compensation charges from the semiconductor and in turn a reduced polarization. This polarization loss would directly affect the $\mathrm{ON}$-current, decreasing for each consecutive cycle, similarly to what is observed in Figure6A.

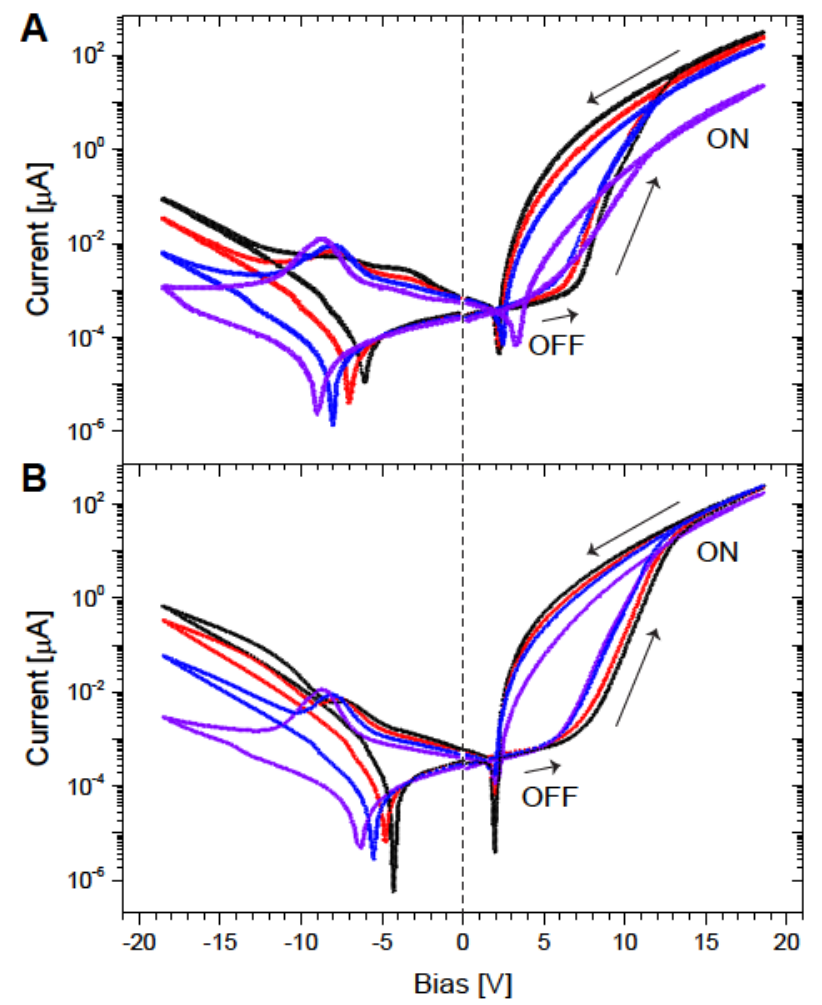

Figure 6. I-V characteristics of the reversible ON- and OFF- switching of: A.BaAl/P(VDFTrFE):F8BT/Au diode and B. BaAl/P(VDF-TrFE):F8BT/MoO ${ }_{x} /$ Mo diode. Arrows indicate the cycle direction. Black, red, blue and purple curves correspond to 1, 10, 100 and 1000 consecutive program-erase cycles respectively.

\section{Conclusion}

A tremendous research effort is currently made in organic electronics and towards the development of non-volatile memory technology. The key requirements are data storage density, read/write speed, ON/OFF ratio, endurance and retention time [1]. To achieve those 
requirements, the assessment of the inter-dependent morphological, chemical and electronic properties within the exploration of new materials, fabrication routes and diode design would ideally require correlated local characterization within a multidisciplinary instrumentation panel with resolution down to the nanometer-scale. Here, undesired crescent-like structures in polymer-based ferroelectric resistive switch diodes were investigated with a panel of different scanning force and $\mathrm{X}$-ray microscopies. These structures were identified to consist of extra-semiconducting material that, in spite of the non-favorable surface energy, wet the $\mathrm{Au}$ substrate due to coordinative interaction during polymer blend phase separation. Associated to these structures, a significant lowering of the ferroelectric polarizibility and degraded device endurance were observed. Alternative diode designs including Au passivation and the use of $\mathrm{MoO}_{x}$ electrode were demonstrated to alleviate the formation of these undesired structures. With the $\mathrm{MoO}_{x}$-based diode, a large increase of the semiconducting channels density and a substantial improvement of the diode endurance were demonstrated, two of the most stringent requirements according to Scott et al [1] . More generally, the work presented here demonstrates the necessity of a multidisciplinary approach to intimately link the material properties at the nanometer scale with the final device performances. As such, this approach constitutes a crucial asset for the further refinement of complex organic electronic devices.

\section{Acknowledgements}

The funding from the European Communitys Seventh Framework Programme (FP7/20072013) under grant agreement no. 248092 of the MOMA project is acknowledged. The Eindhoven University of Technology, PSI (beam time proposal 20130387) and Empa are acknowledged for their respective support. 
Study of the morphology of organic ferroelectric diodes with combined SFM and STXM

\section{References}

[1] J. C. Scott and L. D. Bozano, "Nonvolatile Memory Elements Based on Organic Materials," Advanced Materials, vol. 19, pp. 1452-1463, jun 2007.

[2] K. Asadi, D. M. de Leeuw, B. de Boer, and P. W. M. Blom, "Organic non-volatile memories from ferroelectric phase-separated blends," Nature Materials, vol. 7, pp. 547-550, jul 2008.

[3] M. Kemerink, K. Asadi, P. W. M. Blom, and D. M. De Leeuw, "The operational mechanism of ferroelectricdriven organic resistive switches," Organic Electronics, vol. 13, no. 1, pp. 147-152, 2012.

[4] J. Lee, A. J. J. M. V. Breemen, V. Khikhlovskyi, M. Kemerink, R. A. J. Janssen, and G. H. Gelinck, "Pulsemodulated multilevel data storage in an organic ferroelectric resistive memory diode," Scientific Reports, no. March, pp. 1-7, 2016.

[5] K. Asadi, P. W. M. Blom, and D. M. De Leeuw, "The MEMOLED: Active addressing with passive driving," Advanced Materials, vol. 23, pp. 865-868, 2011.

[6] A. J. J. M. V. Breemen, J.-l. V. D. Steen, G. V. Heck, R. Wang, V. Khikhlovskyi, M. Kemerink, and G. H. Gelinck, "Crossbar arrays of nonvolatile, rewritable polymer ferroelectric diode memories on plastic substrates," Applied Physics Express, vol. 7, p. 031602, 2014.

[7] S. H. Sung and B. W. Boudouris, "Systematic Control of the Nanostructure of SemiconductingFerroelectric Polymer Composites in Thin Film Memory Devices," ACS Macro Letters, vol. 4, pp. 293 297, mar 2015.

[8] A. van Breemen, T. Zaba, V. Khikhlovskyi, J. Michels, R. Janssen, M. Kemerink, and G. Gelinck, "Surface Directed Phase Separation of Semiconductor Ferroelectric Polymer Blends and their Use in Non-Volatile Memories," Advanced Functional Materials, vol. 25, pp. 278-286, jan 2015.

[9] C. R. McNeill, K. Asadi, B. Watts, P. W. M. Blom, and D. M. de Leeuw, "Structure of Phase-Separated Ferroelectric/Semiconducting Polymer Blends for Organic Non-volatile Memories," Small, vol. 6, pp. 508-512, feb 2010.

[10] M. A. Khan, U. S. Bhansali, D. Cha, and H. N. Alshareef, "All-polymer bistable resistive memory device based on nanoscale phase-separated PCBM-ferroelectric blends," Advanced Functional Materials, vol. 23, no. 17, pp. 2145-2152, 2013.

[11] G. M. Su, E. Lim, E. J. Kramer, and M. L. Chabinyc, "Phase Separated Morphology of FerroelectricSemiconductor Polymer Blends Probed by Synchrotron X-ray Methods," Macromolecules, vol. 48, pp. 5861-5867, aug 2015.

[12] V. Khikhlovskyi, A. J. J. M. van Breemen, J. J. Michels, R. A. J. Janssen, G. H. Gelinck, and M. Kemerink, "3D-morphology reconstruction of nanoscale phase-separation in polymer memory blends," Journal of Polymer Science Part B: Polymer Physics, vol. 53, pp. 1231-1237, sep 2015.

[13] J. Hu, J. Zhang, Z. Fu, J. Weng, W. Chen, S. Ding, Y. Jiang, and G. Zhu, "Fabrication of Electrically Bistable Organic Semiconducting/ Ferroelectric Blend Films by Temperature Controlled Spin Coating," Applied Materials \& Interfaces, vol. 7, pp. 6325-6330, 2015.

[14] T. van Woudenbergh, J. Wildeman, P. W. M. Blom, J. J. A. M. Bastiaansen, and B. M. W. LangeveldVos, "Electron-Enhanced Hole Injection in Blue Polyfluorene-Based Polymer Light-Emitting Diodes," Advanced Functional Materials, vol. 14, pp. 677-683, jul 2004.

[15] N. Miyaura and A. Suzuki, "Palladium-catalyzed cross-coupling reactions of organoboron compounds," Chemical reviews, vol. 95, no. 7, pp. 2457-2483, 1995.

[16] N. Pilet, J. Raabe, S. E. Stevenson, S. Romer, L. Bernard, C. R. Mcneill, R. H. Fink, H. J. Hug, and C. Quitmann, "Nanostructure characterization by a combined x-ray absorption / scanning force microscopy system," Nanotechnology, vol. 23, p. 475708, 2012.

[17] V. Khikhlovskyi, R. Wang, A. J. J. M. van Breemen, G. H. Gelinck, R. a. J. Janssen, and M. Kemerink, "Nanoscale Organic Ferroelectric Resistive Switches," The Journal of Physical Chemistry C, vol. 118, pp. 3305-3312, feb 2014.

[18] T. Furukawa, "Ferroelectric properties of vinylidene fluoride copolymers," Phase Transitions, vol. 18, pp. 143-211, 1989.

[19] T. Fukuma, K. Kobayashi, T. Horiuchi, H. Yamada, and K. Matsushige, "Nanometer-Scale Characterization 
of Ferroelectric Polymer Thin Films by Variable- Temperature Atomic Force Microscopy NanometerScale Characterization of Ferroelectric Polymer Thin Films by Variable-Temperature Atomic Force Microscopy," Jpn Journal of Applied Physics, vol. 39, pp. 3830-3833, 2000.

[20] B. Watts, P. Warnicke, N. Pilet, and J. Raabe, "Nanoscale measurement of the absolute mass density of polymers," Physica Status Solidi (A), vol. 212, no. 3, pp. 518-522, 2015.

[21] a. Navid, C. S. Lynch, and L. Pilon, "Purified and porous poly(vinylidene fluoride-trifluoroethylene) thin films for pyroelectric infrared sensing and energy harvesting," Smart Materials and Structures, vol. 19, p. 055006, 2010.

[22] C. M. Björström, S. Nilsson, A. Bernasik, A. Budkowski, M. Andersson, K. O. Magnusson, and E. Moons, "Vertical phase separation in spin-coated films of a low bandgap polyfluorene/PCBM blend-Effects of specific substrate interaction," Applied Surface Science, vol. 253, no. 8, pp. 3906-3912, 2007.

[23] P. Güthner and K. Dransfeld, "Local poling of ferroelectric polymers by scanning force microscopy," Applied Physics Letters, vol. 61, no. 9, pp. 1137-1139, 1992.

[24] V. Khikhlovskyi, A. V. Gorbunov, A. J. J. M. Van Breemen, R. A. J. Janssen, G. H. Gelinck, and M. Kemerink, "Multi-bit organic ferroelectric memory," Organic Electronics, vol. 14, no. 12, pp. 33993405, 2013.

[25] I. P. Batra, P. Wurfel, and B. D. Silverman, "Phase transition, stability, and depolarization field in ferroelectric thin films," Physical Review B, vol. 8, no. 7, pp. 3257-3265, 1973.

[26] P. Wurfel, I. P. Batra, and J. T. Jacobs, "Polarization Instability in Thin Ferroelectric Films," Physical Review Letters, vol. 30, no. 24, p. 1218, 1973.

[27] P. Wurfel and I. P. Batra, "Depolarization-Field-Induced Instability in Thin Ferroelectric Film-Experiment and Theory," Physical Review B, vol. 8, no. 11, p. 5126, 1973.

[28] M. Kröger, S. Hamwi, J. Meyer, T. Riedl, W. Kowalsky, and A. Kahn, "P-type doping of organic wide band gap materials by transition metal oxides: A case-study on Molybdenum trioxide," Organic Electronics, vol. 10, pp. 932-938, 2009.

[29] D. Y. Kim, J. Subbiah, G. Sarasqueta, F. So, H. Ding, Irfan, and Y. Gao, "The effect of molybdenum oxide interlayer on organic photovoltaic cells," Applied Physics Letters, vol. 95, p. 093304, 2009.

[30] T. Putzeys and M. Wübbenhorst, "Asymmetric polarization and hysteresis behaviour in ferroelectric P(VDFTrFE) (76:24) copolymer thin films spatially resolved via LIMM," Phys. Chem. Chem. Phys., vol. 17, no. 12, pp. 7767-7774, 2015.

[31] N. Pilet, V. Khikhlovskyi, A. van Breemen, R. Janssen, M. Kemerink, G. Gelinck, P. Warnicke, and L. Bernard, "Piezoelectricity enhancement of P ( VDF/TrFE ) by X-ray irradiation," Organic Electronics, vol. 37, pp. 257-262, 2016.

[32] D. Guo, I. Stolichnov, and N. Setter, "Thermally induced cooperative molecular reorientation and nanoscale polarization switching behaviors of ultrathin poly(vinylidene fluoride-trifluoroethylene) films," Journal of Physical Chemistry B, vol. 115, no. 46, pp. 13455-13466, 2011.

[33] I. Katsouras, K. Asadi, M. Li, T. B. van Driel, K. S. Kjær, D. Zhao, T. Lenz, Y. Gu, P. W. M. Blom, D. Damjanovic, M. M. Nielsen, and D. M. de Leeuw, "The negative piezoelectric effect of the ferroelectric polymer poly(vinylidene fluoride)," Nature Materials, vol. 15, no. 27, pp. 78-84, 2016.

[34] M. Li, H. J. Wondergem, M.-J. Spijkman, K. Asadi, I. Katsouras, P. W. M. Blom, and D. M. de Leeuw, "Revisiting the $\delta$-phase of poly(vinylidene fluoride) for solution-processed ferroelectric thin films.," Nature materials, vol. 12, no. 5, pp. 433-8, 2013. 